\title{
Multi-Criterial Assessment of the Uniformity of the Electrical Potential of Micro-Films
}

\author{
Horia-Nicolai Teodorescu ${ }^{1,2}$ \\ 1 'Gheorghe Asachi' Technical University of Iasi, \\ Romania \\ ${ }^{2}$ Institute of Computer Science, Romanian \\ Academy, Iasi, \\ Romania
}

\begin{abstract}
We propose a method of characterization and assessment of the uniformity of the surface properties of samples based on a set of derived parameters. Local (sliding window) variance, Allan and Hadamard variances and centers of potential for measurement lines are used to assess the uniformity. The method is exemplified for the potential of piezoelectric thin (nano) films, but it is applicable to a large range of properties.
\end{abstract}

Keywords-uniformity assessment; surface properties; correlational analysis; Allan and Hadamard variances.

\section{INTRODUCTION}

In many unrelated applications, such as sets of synchronized clocks in microsystems, networks of clocks, clocks in GPS systems, uniformity of coverings of surfaces, surface rugosity (roughness), surface charging, and voice pathology, one needs to assess the constancy (uniformity, steadiness) of the variable(s) of interest along the different realizations. The variable of interest may be frequency (clock frequencies, voice specific frequencies), color or thickness of the surface covering, roughness (height of surface irregularities), or charge in case of charged surfaces. The 'realizations' may be the different frequency recordings, one per clock (object), the series of thickness values measured along lines of samples of covered material, the values of color of the pixels along columns of images, or the values along different lines of measurement of the charge (or potential) along surfaces of a set of dielectric samples.

Various specialties tend to use different parameters in assessing the uniformity. For example, clock frequency steadiness is often characterized using Allan and Hadamard variances [1-5], surface roughness in tribology is defined in terms of maximal "peak" and "valley" dimensions (i.e., range interval) and of the standard deviation of the height of the surface elements $\left(R_{a}, R_{q}, R_{z}\right.$, see [6]), color uniformity is sometimes assessed using descriptive statistics and Fourier analysis, while individual studies in voice pathology may use a combination of all the above as well as supplementary methods and indices such as those based on the correlation [7 HNT].

\author{
Victor Cojocaru ${ }^{3}$ \\ Institute of Electronic Engineering and \\ Nanotechnology, Academy of Sciences of \\ Moldova, Chisinau 2028, Moldova \\ Alexei Katashev ${ }^{4}$ \\ ${ }^{2}$ Riga Technical University, Biomedical \\ Engineering and Nanotechnologies Institute, Riga, \\ Latvia
}

In applications related to sensors, micro-actuators, and electricity micro-generators (energy harvesting), the surface uniformity of the properties of piezoelectric films is important for accuracy and for reproducibility in serial manufacturing. We propose a set of uniformity indices that refine the picture offered by basic statistical descriptors. Some of these indicators of uniformity are directly derived from common electrical quantities; others are adopted from unrelated disciplines.

We propose indicators of uniformity derived from common electrical quantities: (a) the center of variable potential (defined similarly to the center of charge or mass) for the entire sample, or measurement line(s), when the sample has an axis of symmetry, moreover (b) the same for each half of the sample or measurement line; (c) the maximal and average local electric fields (local derivative of the potential), and the standard deviation of the local electric field; (d) the local standard deviation and its center. The indices of uniformity borrowed from other disciplines are Allan and Hadamard variations of the potential and their centers; to these, one may add indices based on the self-correlation function of the potential along a measured line [7], but we do not discuss these here.

Although in this article we use the piezoelectric films as a suitable case for exemplifying the method, the same scheme is applicable to many other situations, such as roughness characterization.

\section{JUSTIFICATION OF THE MULTI-CRITERIAL ASSESSMENT FRAMEWORK}

While some basic applications with low quality requirements may be satisfactory serviced with a simple statistical analysis including just a few parameters, such as standard deviation and range of the supposedly steady variable, higher end applications may require a refined, detailed approach. As a matter of example, the requirements for the uniformity of the electrical charging of a fabric for dust filters can be much lower than the charge uniformity asked for membranes in electret microphones. 
In what follows, we consider that a single quantity (variable) represent the set of objects to be assessed. Consider that the realizations $\left\{\boldsymbol{x}^{h}\right\}, h=1, \ldots, n$, have all a number of $N$ measurement values, $x_{k}^{h}, k=$ $1, \ldots, N$. In the first place, the use of the statistics over an entire set of realizations should be replaced with the comparison of the statistics over individual realizations (comparison of the local statistics). This may be less evident when the problem is to characterize the uniformity of the color or the roughness, but it becomes clear when the realizations are the signals of distributed clocks. In this respect, for gaining insight, the standard deviation of the overall population of measurements, $\sigma_{\text {all }}$, should be replaced with the vector of the local deviations, $\left\{\sigma_{h}\right\}$, and a vector of averages, $\left\{\mu_{h}\right\}$ should replace the overall average, $\mu$. Here, $\sigma_{h}$ stands for the standard deviation of the set of values of $\boldsymbol{x}^{h}$ and $\mu_{h}$ stand for the average of $\boldsymbol{x}^{h}$. Then, the statistics of the sets $\left\{\mu_{h}\right\}_{h}$ and $\left\{\sigma_{h}\right\}_{h}$ are of interest: it is desirable that the population $\left\{\mu_{h}\right\}_{h}$ has very low standard deviation, to insure uniformity, moreover the range of $\left\{\sigma_{h}\right\}_{h}$ should be as small as possible. The same reasoning goes for the sets of values of Allan and Hadamard variances (AVAR and $H V A R$ ) determined over moving windows, as in [7].

Typically, measurements of 2D samples ('events') are performed along measurement lines, such as those in atomic force microscopy (AFM), roughness, and electric surface charge. We restrict the discussion to this case.

\section{EXAMPLE OF APPLICATION}

In a related research [8, 9], nano films of poly(vinylidene fluoride) (PVDF) were deposited and their 3D height profile and the electric potential map was determined using atomic force microscopy (AFM), as described in [8]. For the method, see [912]. PVDF is widely used in various applications as a piezoelectric material, but it can have very different properties, depending on the film growth procedure [13-16]. Of particular interest is to determine the potential distribution after provoking the piezoelectric effect in regions of the film, using AFM techniques. Figure 1 shows the 3D graph of the potential distribution on the surface of the probe, with a central square of the sample polarized with the AFM in order to determine its piezoelectric behavior.

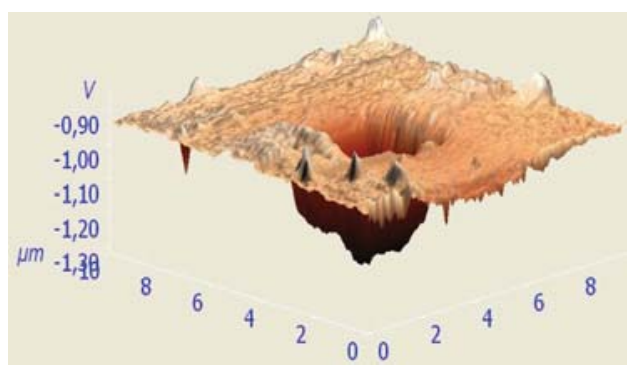

Figure 1. $3 \mathrm{D}$ potential distribution on the sample

A central measurement line of the potential, $u(x)$, is shown in Fig. 2 and constitutes the basis of the discussion in this article.

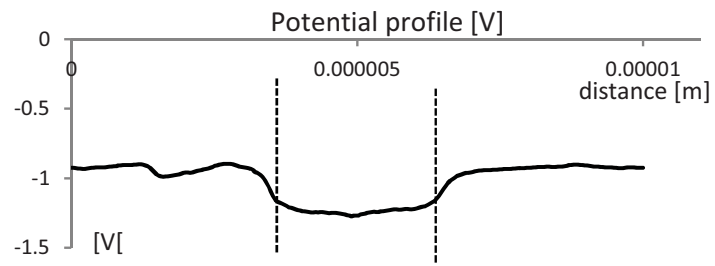

Figure 2. Potential profile; the analyzed region is between the vertical lines

The surface electric field (tangential electric field component along the measurement line), defined as the potential difference between adjacent points, is defined based on the general definition $\Delta \mathrm{u}(x) / \Delta x$, precisely at measurement point $x_{k}$ as $\left(u_{k+1}-\right.$ $\left.u_{k-1}\right) /\left(x_{k+1}-x_{k-1}\right)$. The values of the microscopic electric field are very large because of the small distances involved, see Fig. 3. While the average of the field is relatively low $(49.9 \mathrm{~V})$, that is, the potential gradient is low on average, the standard deviation of the field is very large, $176195 \mathrm{~V}$, indicating a large local variability of the potential gradient along the surface (precisely, along the measurement line).

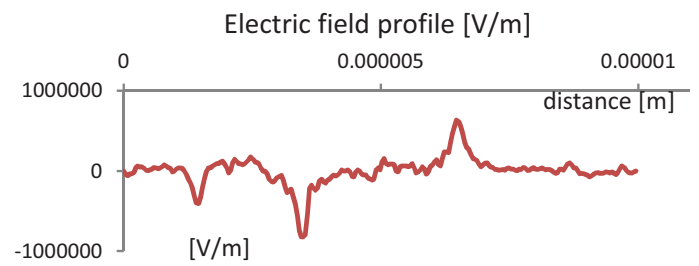

Figure 3. The graph of the electric field numerically dedetrmined (see the text).

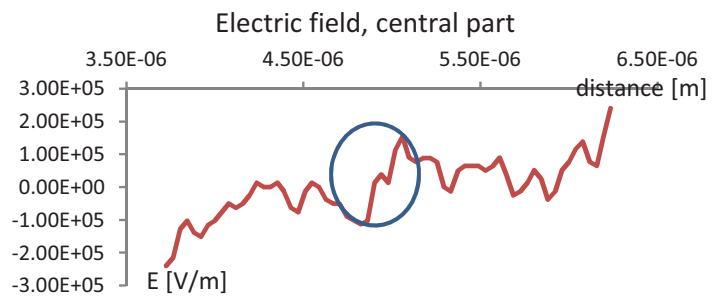

Figure 4. Variation of the electric field

Notice in Fig. 4 that the variations of the electric potential (and field around zero values) produce local extremes that can act as traps for charges with the appropriate charge sign. For example, the local region in the circle in Fig. 4 is around zero electric field, meaning that it acts as a trap for negative charges.

\section{DEFINING LOCAl Allan AND HADAMARD} VARIANCES OF THE POTENTIAL AND THEIR ASYMETRY

Considering the window $W(h, 2 n+1)$ of width $2 n+1$ centered at $n+h+1$, we define the local variance of a signal as [7]

$$
\sigma_{x, W, h, n}^{2}=\frac{1}{2 n+1} \sum_{k=h-n}^{h+n}\left(x_{k}-\bar{x}_{W}\right)^{2}
$$

where $\bar{x}_{W}$ is the average on the window $W$. Allan variance, denoted by $A V A R$, is defined similarly, but using the derived sequence [1-5],

$$
y_{k}=x_{k}-2 x_{(k-1)}+x_{(k-2)} .
$$

$\operatorname{AVAR}\left(x_{W}\right)$ is the variance of $\left\{y_{k}\right\}$, 


$$
\operatorname{AVAR}\left(x_{W}\right)=\frac{1}{n-1} \sum_{k=h+2}^{n-1}\left(y_{k}-\bar{y}_{W}\right)^{2} .
$$

The local Hadamard variance, denoted by HVAR or $\sigma_{H 0}^{2}$, is defined based on the derived sequence

$$
z_{k}=x_{k}-3 x_{(k-1)}+3 x_{(k-2)}-x_{(k-3)} .
$$

Then, the Hadamard variance with null displacement, $H V A R$, is the variance of the series $\left\{z_{k}\right\}$. Allan and Hadamard variances are largely used in frequency stability analysis, see [1-5]. Local standard deviation (STDEV), defined as above for sliding windows, and local $A V A R$ and $H V A R$ series were computed on moving windows of 40 samples, with the window having 19 predecessor samples and 20 successors, starting at the $20^{\text {th }}$ sample and ending 20 samples before the end of the series. The series of the local standard deviation computed on the same windows is shown in Fig. 5. Notice that the local STDEV for the potential is much larger than $A V A R$ and HVAR (Fig. 6), but shows less details of the uniformity. The local standard deviation is maximal, as expected, at the border of the sample, where the potential is less uniform.

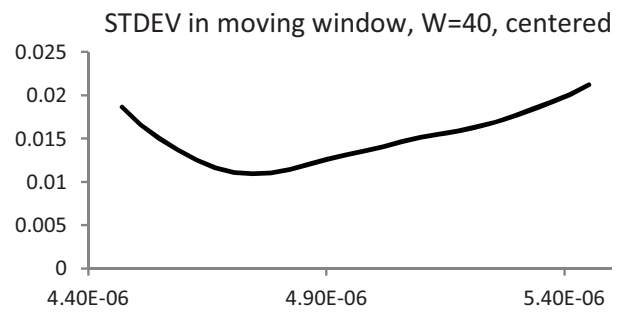

Figure 5. Standard deviation in the central part of the measurement line

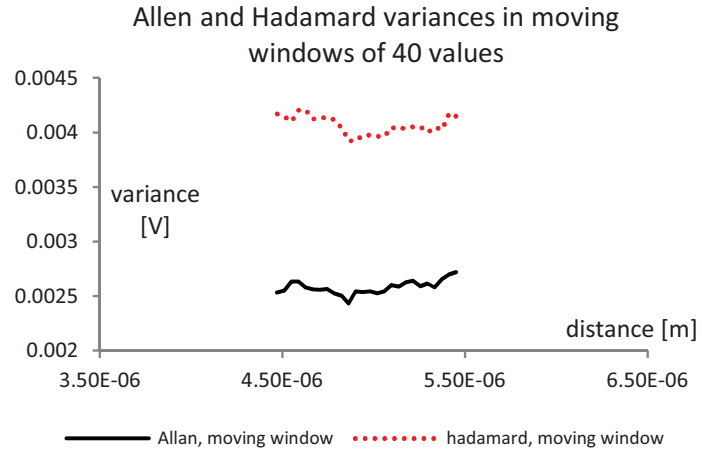

Figure 6. Allen and Hadamard variances in moving windows of 40 values

Figures 5 and 6 show that all three (standard, Allan, and Hadamard) series of local variances look asymmetric.

\section{CENTER OF POTENTIAL AND ASYMMETRIES ALONG MEASUREMENT LINES}

The notion of center of a linearly distributed quantity (charge, mass) is easily extended to the concept of potential, defined for a discrete linear charge distribution as

$$
x_{c U}=\frac{1}{\sum_{k=1}^{n} u_{k}} \cdot \sum_{k=1}^{n} x_{k} u_{k}
$$

where $n$ is the number of values of potential, $u_{k}$, along a line. For the upper half of the measurement line, the center of potential is defined as

$$
x_{c U-u}=\frac{1}{\sum_{k=1}^{40} u_{k}} \cdot \sum_{k=1}^{40} x_{k} u_{k}
$$

where $x_{k}$ are the coordinates along the measurement line. Similarly is defined the center of charge for the half-lines in the lower half of the sample, $x_{C U-l}$. The difference $\Delta x_{c U}=x_{c U-u}-x_{c U-l}$ is null for symmetrically distributed charges. Large values of $\Delta x_{c U}$ would show asymmetries of the potential.

The ratio to half length of the 'middle potential' for the half upper part is $5.05 \mathrm{E}-01$ (i.e., at $50.5 \%$ of the upper part, only $0.5 \%$ distance from the geometric middle of the upper part). The middle potential of the lower half part is at 5.12E-01 $(1.2 \%$ error from the geometric middle). Therefore, these quantities show a very good symmetry of the potential along the measurement line. From the point of view of these indicators, the potential asymmetry is very low.

Applying the same concept of center and similar formulas for the three deviations discussed above, we obtain for the regions in Figs. 5 and 6: geometric center at 4.96E-06, center STDEV at 5.00E-06, relative error with respect to the center ( 100 . $\left.\left(x_{S D T V}-x_{\text {true }}\right) /(\max x-\min x)\right), 3.55 \%$, center Allan variance at $4.96 \mathrm{E}-06$, error $0.4 \%$, center of Hadamard variance at $4.96 \mathrm{E}-06$, error $-0.2 \%$. Therefore, the local STDEV variance is in this case more indicative of the asymmetry.

\section{POTENTIAL AND ELECTRIC FORCE ASYMMETRIES}

The potential and forces created by the charge distribution at the center of the sample are important in applications. Assume that the measured local potentials are proportional with the local surface charges. In case of perfect symmetry, the potential at the center of the measurement line (line of charge), as created by the left and right sides, should be null. The value of the potential, at very small distances from the surface, is then given by the relation (which is in a way the reciprocal of the center of potential)

$U_{\text {center }}=\kappa \cdot\left(\sum_{\substack{u=1 \\ \text { up }}}^{40} \frac{u_{k}}{x_{\text {center }}-x_{k}}-\sum_{k=1}^{40} \frac{u_{k}}{x_{k}-x_{\text {center }}}\right)$.

Computing for the region of interest, the difference in potential between the upper and lower halves of the line is less than $1 \%$ (upper/lower ratio is 1.0023 ). This result shows again a good symmetry. Above, $\kappa$ is a constant; it is irrelevant in this discussion.

The force along the line (tangential force) produced at the center of the line is computed with a similar formula (using squares at the denominator, $\left.\left(x_{k}-x_{\text {center }}\right)^{2}\right)$.

\section{DISCUSSION AND CONCLUSIONS}

The summary of the results presented in Table I shows that the variation of the potential is small, with the standard deviation representing less than $1.6 \%$ of the average, that the overall symmetry of the potential is good, with the center of the potential very close to the geometric center (4.978E-06 vs. 4.980E-06), 
moreover that even the two halves of the measuring line exhibit almost perfect symmetry in terms of their respective centers of potential. The overall variation of the derivative $(A V A R)$ is smaller than the standard deviation ( 0.0025 vs. 0.0195$)$, which means that the variations are not very fast; the overall $H V A R$ is also small, also larger than AVAR. Yet, the detailed analysis performed with sliding window standard deviation, AVAR and HVAR show that the local variations are not perfectly symmetrical, see Figs. 5 and 6.

TABLE I. SUMMARY OF THE RESULTS FOR THE ASYMMETRY OF THE POTENTIAL, FOR THE CENTRAL PART OF THE SAMPLE

\begin{tabular}{|l|r|}
\hline Average potential [V] & -1.237 \\
\hline STDEV (total) [V] & 0.0195 \\
\hline Center (Middle) of potential [m] & $4.978 \mathrm{E}-06$ \\
\hline Geometric middle of the measured region [m] & $4.980 \mathrm{E}-06$ \\
\hline Middle potential upper part [m] & $4.358 \mathrm{E}-06$ \\
\hline Middle potential lower part [m] & $5.623 \mathrm{E}-06$ \\
\hline Distance (length of measured region) [m] & $2.510 \mathrm{E}-06$ \\
\hline STDEV/aver \% & -1.580 \\
\hline Error of the center of potential for the upper part & $0.5 \%$ \\
\hline Error of the center of potential for the lower part & $1.2 \%$ \\
\hline Min potential [V] & -1.273 \\
\hline Max potential [V] & -1.192 \\
\hline max-min potential [V] & 0.081 \\
\hline AVAR (overall) [V] & 0.0025 \\
\hline HVAR (overall) [V] & 0.0039 \\
\hline
\end{tabular}

In this paper we proposed the use of a multicriterial assessment framework that combines several methods from different fields. The proposed method of assessing uniformity combines descriptive statistics with spatially weighted averages, and Allan and Hadamard variances; correlational analysis may supplement the analysis. We exemplified the framework for an application (PVDF piezo nano film) and discussed the benefits of the framework.

The results of applying the methodology to a case of potential induced by piezoelectric effect in a nano film of PVDF shown a good uniformity of the potential, and detailed limits of the symmetry of the potential along a measurement line.

Future research will be guided to apply the methodology presented in [7] and in this paper to other cases of charged surfaces and to the characterization of the roughness of the surfaces.

While AFM microscopes are typically accompanied by powerful software packages for processing the results, these packages are not provided with means specific for assessing the uniformity of the surface properties. We suggest that it would be useful to include, in the software packages of AFM and other microscopes and devices for the analysis of surfaces, the means to assess the uniformity as proposed in this paper.

\section{ACKNOWLEDGMENT}

HNT thanks Prof. L. Dascalescu on a comment on an aspect of the method.
Authors' contributions: HNT proposed the concepts (partly in a previous paper), proposed the topic and the plan of the research, processed the data, and wrote the initial version of the paper. $\mathrm{VC}$ and $\mathrm{AK}$ performed numerous measurements (not all reported in this paper) and collected the data. All authors agreed with the final form of the paper. The authors declare no conflict of interest.

\section{REFERENCES}

[1] D. Howe, R. Beard, C. Greenhall, F. Vernotte, B. Riley, "Total Hadamard Variance: application to clock steering by Kalman filtering." http://citeseerx.ist.psu.edu/viewdoc/ download?doi=10.1.1.598.1541\&rep=rep1\&type=pdf.

[2] N. Ashby, B. Patla, "Simulations of the Hadamard variance: probability distributions and confidence intervals." IEEE Trans. Ultrasonics, Ferroelectrics, and Frequency Control, Vol. 63, 4, Apr 2016, pp. 636-645.

[3] W.J. Riley, Handbook of Frequency Stability Analysis. NIST Publication 1065, Nat. Inst. Standards and Tech. Boulder, CO USA, July 2008, https://tf.nist.gov/general/pdf/2220.pdf.

[4] J.A. Barnes et al., "Characterization of frequency stability." IEEE Trans. Instruments and Meas. 20, pp. 105-120, 1971.

[5] S.T. Hutsell, W.G. Reid, J.D. Crum, H.S. Mobbs, J.A. Buisson, "Operational use of the Hadamard variance in GPS. Report", U.S. Naval Observatory, CO., Dec. 1996, https://apps.dtic.mil/dtic/tr/fulltext/u2/a516917.pdf.

[6] T.V. Vorburger, J. Raja, "Surface Finish Metrology Tutorial", NISTIR, 89-4088, Dept of Commerce, USA, 1990, https://www.nist.gov/sites/default/files/documents/calibration s/89-4088.pdf.

[7] H-N. Teodorescu, "Using local variance, Allan- and Hadamard variances in speech analysis-Pitch analysis." Proc. IEEE 14-th International Symposium on Signals, Circuits and Systems. ISSCS 2019, Iasi, Romania, July 11 - 12, 2019.

[8] V. Cojocaru, A. Katashev, H.-N. Teodorescu, "Analysis of the behavior of PVDF nano-layers deposited under various conditions." Int. Conf. Nanotech. \& Biomedical Engineering, Chişinău, R. Moldova, 7-8 July, 2011, pp. 80-82, available at http://www.sibm.md/uploads/files/BIONANOCONF2011.pdf accessed April 2, 2019.

[9] A. Katashev, V. Cojocaru, M. Hulea, H.N. Teodorescu, Metoda de realizare a nano- si micro- filmelor piezoelectrice PVDF prin racire de la temperaturi ridicate in camp electric. (in Romanian) https://www.imt.ro/NANOPROSPECT/postere expo/Victor COJOCARU_Poster_HNTeodorescu_UTIASI(P) ROSPECT_Bucuresti_2011.pdf. Poster, NANOPROSPECT, Bucharest $\overline{24}$ mai $201 \overline{1}$.

[10] A. Batagiannis, M. Wübbenhorst, J. Hulliger, "Piezo- and pyroelectric microscopy", Current Opinion in Solid State and Materials Sci, Vol. 14, Issue 5, Oct. 2010, pp 107-115.

[11] M. Alexe, A. Gruverman (Eds.). Nanoscale Characterisation of Ferroelectric Materials. Scanning Probe Microscopy Approach. Springer, 2004.

[12] W. Melitz, J. Shena, A.C. Kummel, S. Lee, "Kelvin probe force microscopy and its application." Surface Science Reports 66 (2011) 1-27.

[13] W. Ma, J. Zhang, X. Wang, "Formation of poly(vinylidene fluoride) crystalline phases from tetrahydrofuran/N, Ndimethylformamide mixed solvent." J Mater Sci (2008) 43:398-401.

[14] T. Jee, H. Lee, B. Mika and H. Liang, "Effect of microstructures of PVDF on surface adhesive forces." Tribology Letters, Vol. 26, No. 2, May 2007.

[15] S. Mani, R. Perez, H. Lee, Z. Ounaies, W. Hung and H. Liang, "Effects of applied potential on friction of a piezoelectric material." J. Tribol, Oct 2007, Vol 129, 4, pp. 836-840.

[16] V. Ostaševičius, I. Milašauskaitè, R. Daukševičius, V. Baltrušaitis, V. Grigaliūnas, I. Prosyčevas, "Experimental characterization of material structure of piezoelectric PVDF polymer." Mechanika. 2010. Nr. 6 (86), 78-82. 